\title{
BIBLIOGRAFIA
}

\section{Anónimos y Seudónimos Hispanoamericanos}

L único propósito que tuvimos al empezar a reunir estos datos
sobre los catálogos de seudónimos y publicaciones anónimas, fué que una vez reunidos nos servirían de modelo y de guía para formar otro de autores mexicanos. No nos costó poco trabajo localizar en los Esstados Unidos los libros de que damos aquí noticia, porque en primer lugar existen muy pocos ejemplares que hayan llegado hasta acá, y en segundo, porque sólo con paciencia y a veces -confesémoslo francamente- con engaño hemos podido ver estas rarezas en las salas donde catalogan los libros nuestros bibliotecarios. Hay libros de esta clase que eludiendo cartas, ruegos, pesquisas tenaces (aun de los mismos bibliotecarios que juran haberlos tenido siempre a la mano), parecen haber aprendido sus esquivas mañas de la misma paloma de la paz. Sin embargo, tendiéndoles trampas de una especie u otra hemos logrado verr la mayoría y darnos cuenta de que se habian empleado en la sistematización de las fichas tantos métodos como había bibliógrafos. El estudio de tales catálogos no satisfizo completamente nuestro primer propósito, pero sí nos sugirió la conveniencia de elaborar un método que reuniera en sí las varias ventajas que se observaron esparcidas en los distintos catálogos. Antes de todo, protestamos humildemente que la aportación nuestra no es grande u original y que al proponer este método sólo hemos intentado valernos lo más posible de la experiencia práctica que viene del estudio de los catálogos ya publicados. 
Ofrecemos, pues, este plan de trabajo a varios colegas que nos han avisado que preparan estudios de la misma índole, por si acaso encuentran en él algo provechoso, y en espera de unificar las labores que en distintos lugares llevamos adelante hacia el mismo fin. Que sirva también este método propuesto como medida, desde nuestro punto de vista, de la utilidad relativa de los catálogos que describimos en seguida de esta advertencia. Nos limitamos abajo a hacer una descripción de cada uno, bien enterados de las dificultades que confrontaron los autores. Por muchos que sean los defectos que se observan en algunos de tales catálogos - hay otros que son intachables-, no dejan de merecer el elogio sincero de los bibliotecarios, bibliógrafos y críticos que necesariamente acuden a la generosa ayuda que les proporcionan los compiladores.

El método que ofrecemos para la elaboración de un catálogo de seudónimos y anónimos es el que sigure. Debemos decir desde luego que sería deseable limitar el catálogo a un país y tener para cada país iberoamericano un inventario de seudónimos y publicaciones anónimas de autores nacionales y extranjeros que publicaron sus obras en él. Esstos estudios se dividen, por su naturaleza, en dos obras distintas. La primera consiste, en nombres falsos e iniciales que ocultan los nombres propios de autores que publican sus producciones literarias en periódicos o en revistas; la otra, en publicaciones anónimas o seudónimas dadas a luz en forma de libro o folleto. Parece más útil a quien busque el verdadero apellido del periodista o literato encontrarlo en una sola lista de seudónimos numerados y puestos en orden rigurosamente alfabético, letra por letra. En esta lista, al seudónimo siguen el nombre del autor, las fechas del nacimiento y de la muerte, los nombres de los periódicos o revistas en que publicó sus artículos y los periodos durante los cuales se sirvió del seudónimo. Además, para hacer tal lista aún más útil, bueno seria agregar unos pocos datos biográficos y la citación exacta de los artículos publicados, con fecha, lugar de impresión y paginación. Si tiene cabida, se puede comprobar la identidad del autor haciendo constar las razones que conducen a creerlo, o cuando menos las autoridades que lo afirman. Al fin de este diccionario de seudónimos debe ponerse un registro de apellidos de autores, con referencia por números a la sección de seudónimos. 
Ya queda bien establecida la sistematización de publicaciones anónimas o seudónimas, puesto que se acostumbra ponerlas por orden estricto de los títulos numerados. Sigue el nombre propio del autor y los medios de que se sirvió el bibliógrafo para descubrirle. Si se dispone del espacio necesario para hacerlo, sería provechoso añadir datos biográficos del autor, y bibliográficos de las otras ediciones, si las hay. Al fin deben hallarbe, cuando menos, dos índices: uno de apellidos de los autores, otro de seudónimos o iniciales. En ellos se haría referencia a los títulos por medio de los números que éstos tienen. Siguiendo tal procedimiento serán servidas las necesidades más generales de los bibliotecarios, bibliógrafos y críticos. En caso de que se quiera formar un catálogo de mayor alcance, existe excelente modelo en la obra de Uriarte (véase núm. 34).

Hemos incluído catálogos españoles y portugueses por dos razones: 1) contienen también referencias a autores iberoamericanos, y 2) muchos seudónimos de autores de los dos países aparecen en artículos y libros reimpresos en Iberoamérica.

\section{INDICE DE LOS CATALOGOS POR PAISES}

I. General. . . ......núms. 3, 4, 7, 11, 19, 34, 35, 36 .

Brasil. ............. 9, 24.

Colombia........... s, 15 .

Cuba............. 8, 10.

Chile. . . . . . . 16,17 .

Ecuador. . . . 27.3.

México........... 12, 13.

El Perú. . ......... 1, 6, 22.

El Uruguay. .......... $32,33$.

II. Obras inéditas o en preparación

General. . . . ......... 18, 20, 23, 26.

Costa Rica. . . . . . . 37.

Guatemala. . . ........ 2.

México........... 14, 21 .

Rep. Dominicana. . . . . 25. 


\section{ABREVIATURAS.}

Empleamos los símbolos establecidos por el Catálogo de la Unión, Washington, D. C., para indicar las bibliotecas que poseen ejemplares de las revistas y los libros mencionados en esta bibliografía.

$\mathrm{AzU}$

Biblioteca de la Universidad de Arizona, Tucson, Arizona.

CSmH Biblioteca Henry Huntington, San Marino, California.

Ct Biblioteca del Estado, Hartford, Connecticut.

CU Biblioteca de la Universidad de California, Los Angeles, California.

DCU-IA Colección Iberoamericana de la Universidad Católica, Washington, D. C.

DLC Biblioteca del Congreso, Washington, D. C.

DPU Biblioteca de la Unión Panamericana, Washington, D. C.

ICJ Biblioteca John Crearer, Chicago, Illinois.

ICU Biblioteca de la Universidad de Chicago, Chicago, Illinois.

IU

Biblioteca de la Universidad de Illinois, Urbana, Illinois.

MB : Biblioteca Pública de Boston, Boston, Massachusetts.

MH Biblioteca de $l_{2}$ Universidad de Harvard, Cambridge, Massachusetts.

MWA Biblioteca de la American Antiquarian Society, Worcester, Massachusetts.

NcD Biblioteca de la Universidad de Duke, Durham, North Carolina.

NcU Biblioteca de la Universidad de North Carolina, Chapel Hill, North Carolina.

NjP Biblioteca de la Universidad de Princeton, Princeton, Nueva Jersey.

NN Biblioteca Pública de Nueva York, Nueva York, N. Y.

NNC Biblioteca de la Universidad de Columbia, Nueva York, N. Y. .

NNH Biblioteca Huntington, Nueva York, N. Y.

OCU Biblioteca de la Universidad del Estado de Ohio, Columbus, Ohio. 
PBm . Biblioteca de Bryn Mawr College, Bryn Mawr Pennsylvania.

PU Biblioteca de la Universidad de Pennsylvania, Philadelphia, Pennsylvania.

PU-M Biblioteca del Museo de $1_{a}$ Universidad de Pennsylvania, Philadelphia, Pennsylvania.

RPJCB Biblioteca John Carter Brown, Providence, Rhode Island.

RPB Biblioteca de la Universidad de Btown, Providence, Rhode Island.

TxU Biblioteca de la Universidad de Texas, Austin, Texas.

WaU Biblioteca de la Universidad de Washington, Seattle, Washington.

ANónIMO.

1. "Para un catálogo de seudónimos peruanos". Boletin Bibliográfico publicado por la Biblioteca Central de la Universidad [Mayor de San Marcos] (Lima, Perú), VIII, 2 (julio 1938), 185-188.

Esta primera compilación de seudónimos de autores peruanos consiste en dos listas. La primera contiene 21 seudónimos "en libros". la segunda, 97 "en periódicos y revistas", puestas en orden alfabético. El apellido del autor sigue al seudónimo sin la citaciọ́n del libro o de la revista. Ejemplares: NcU/DLC.

ArÉValo Martínez, RaFaÉr.

2. En contestación a nuestra consulta nos informa este ilustre autor que en Guatemala hasta la fecha "ninguno se ha dedicado a la elaboración de catálogos y obras donde se haga - una sistematización de los pseudónimos y anónimos". Gestiona la compilación de uno.

Barros Arana, Diego.

3. Notas para una bibliografía de obras anómimas $i$ sendónimas sobre la historia, la jeografía $i$ la literatura de Antérica. Santiago de Chile, Imprenta Nacional, 1882.171 pp. $26 \mathrm{~cm}$. 
Tirada aparte de Anales de la Universidad de Chile, LXI (1882), pp. 5-171. A tan fecundo escritor se debe el primer catálogo dedicado a esta materia por antor iberoamericano. Sin limitación de lagar de impresión, hace referencia a 507 títulos de libros que tratan de las tres Américas. Consta de dos partes. En la primera, que cataloga las obras por orden alfabético de títulos numerados. "ponemos - dice él- el nombre del autor i de ordinario las noticias que acerca de éste o de su libro puedan interesar más directamente al lector. En la segunda parte, catalogamos a los autores por orden alfabético, indicando mui sumariamente sus obras anónimas, $i$ baciendo una referencia numérica a la nota que le corresponde en la primera sección". Prefacio. Véase la obra de José Toribio Medina mencionada abajo. El método empleado por Barros Arana se adapta mny bien al asunto y los datos que proporciona son por lo general fidedignos y útiles.

Ejemplares: DLC/ICJ/MH/MWA/NN/OCU/PU-M

4. Idem. Obras completas de Diego Barros Arana. Tomo VI. Estudios histórico-bibliográficos. Santiago de Chile, Imprenta Cervantes, 1909. Páginas 369-555.

Reimpresión de número tres.

Ejemplares: CU/DCU-IA/DLC/IU/MB/NcU/NjP/NN/PBm/ PU

Cabral,es, Simón D.

5. Catálogo de seudónimos colombianos. E1 Monitor, antes de 1882.

Isidoro Laverde Amaya (q. v.) bace una referencia a esta obra, agregando: "lo hemos completado a fin de que sirva de guía de los nombres de los autores a los curiosos que quieran hojear las páginas de nuestros numerosos periódicos literatios y políticos". p. 237 .

Cornejo Bouroncle, Jorge.

6. "Algunos seudónimos de escritores cuzqueños". Boletin Bibliográfico publicado por la Biblioteca Central de la Universidad Mayor de San Marcos (Lima, Perú), XIII, 1/2 (junio, 1940), 1-4.

Por orden alfabético cataloga 65 seudónimos que los autores cuzqueños empleaban en periódicos nacionales. Además de dar 
el nombre del autor agrega a menudo datos biográficos o críticos y de vez en cuando cita el nombre del periódico en que apareció el artículo seudónimo.

Ejemplares: DLC/DPU/NcU

\section{Fellú Cruz, Guilizermo.}

7. Advertencias saludables a un criticastro de mala ley. Del. Boletín del Instituto de Investigaciones Históricas (de la Facultad de Filosofía y Letras), no. 40, tomo VIII, págs. 254 a 259 y no. 42 , tomo IX, págs. 237 a 280 , correspondientes a los meses de abril/junio y octubre/diciembre de 1929. Buenos Aires, Imprenta de la Universidad, 1929. $56 \mathrm{pp}$.

E1 Conservador de la Sala Medina en la Biblioteca Nacional, Santiago de Chile, impugna la "muestra palpable de una petulancia moy ajena a la cultura de nn verdadero investigador" que ofrece Ricardo Victorica (q. v.) en los dos libros suyos que atacan a José Toribio Medina por haber dejado incompleto y no sin errores su Diccionario de anónimos y seudónimos hispanoamericanos. A la vez que hace una defensa de Medina, Feliú Cruz señala las fuentes que usó Victorica al compilar sus dos libros y crítica con justicia y detalladamente el método y la materia. Las advertencias de Feliú Cruz constan en primer lugar de 19 observaciones sobre Errores y omisiones del Diccionario de anónimos y pseudónimos hispanoamericanos de José Toribio Medina (1928). Después, refiriéndose a Nueva Epanortosis (1929), el defensor de Medina impugna las 33 correcciones que hace Victorica' a la obra de Medina, señala las 18 veces que "Victorica se aprovecha malamente de la obra de Medina" y por fin descubre 64 "errares y dislates de la Nueva Epanortosis". Victorica sin duda perdió esta polémica, no, claro, porque hubiese errores en las obras que compiló, sino porque quedó probado que más le motivó al escribirlos el deseo de zaherir a Medina que el de hacer él mismo una obra más perfecta. No resultó infructuosa la polémica, porque la defensa que hizo Feliú Cruz de Medina resulta ser una fe de erratas muy útil a las obras de Victorica.

Ejemplares: DLC.

Figarola-Caneda, Domingo.

8. Diccionario cubano de setudónimos. Habana, Imprenta "El Siglo XX", 1922. xvi, 182 pp. $25.5 \mathrm{cms}$.

Hay 2.250 citaciones. Consta de dos listas. La primera de iniciales y seudónimos de escritotes cubanos y extranjeros que escri- 
bieron sobre Cuba en los siglos XIX y XX, puestos por orden alfabético. Las más veces da el autor las fechas precisas del nacimiento $y$ de la muerte del autor discutido $y$ hace referencia a varias de sus obras. La segunda lista. es de nombres propios. También contiene un suplemento.

Ejemplares: $\mathrm{CU} / \mathrm{DLC} / \mathrm{DPU} / \mathrm{ICJ} / \mathrm{NjP} / \mathrm{NN}$

Fonseca, Maritinho Augusto Ferreira da.

9. Subsidios para um diccionario de psendonymos, iniciaes $e$ obras anonymas de escriptores portuguezes, contribuição para o estudo da literatura portugueza. Lisboa, Por ordem e na typ. da Academia Real das Sciencias, 1896. xii, 298 pp. 23 cms.

Hay 2,400 citaciones divididas en tres partes, cada una por orden alfabético: 1) de seudónimos $(800)$ de escritores portugueses, sin importar dónde se publicaron sus libros; 2) de iniciales (400); 3) de obras seudónimas y anónimas. Incluye las fechas del nacimiento" y de la muerte de los autores. Al final hay un suplemento con adiciones. Hace falta un indice onomástico refitiéndose a las tres secciones.

Ejemplares: DLC/ICJ/NjP

García Garófalo y Mesa, Manuel.

10. Diccionario de setdónimos de escritores, poetas y periodistas villaclareños. La Habana, Imp. Julio Arroyo, 1926. 61 pp. $17.5 \mathrm{cms}$.

Relativo a autores del pueblo de Santa Clara, en los siglos XIX Y XX. Cataloga 125 seudónimos, dando las fechas del nacimiento $y$ de la muerte, si le son sabidas, más un breve comentario sobre los géneros en que el autor ejercia su pluma. Falta un índice de nombres propios.

[HARTzenbusch É Hiriart, Eugienio].

11. Unos cuantos seudónimos de escritores españoles con susı correspondientes nombres verdaderos. Apuntes recogidos $y$ coleccionados por Maxiriarth [seud.] con un prólogo del Sr. D. José Fernández Bremón. Edición corregida y au-' mentada. Madrid, Estab. Tipográfico Sucesores de Rivadeneyra, 1904. xix, 168 pp. $17.5 \mathrm{cms}$. 
La primera edición, según se ve en el prefacio, la publicó en 1892. La mayoría de los autores mencionados son del siglo pasado. Las fichas, por orden alfabético de seudónimos, indican a veces el año de nacimiento o de muerte del autor y a veces. lás más, el periodo durante el cual escribió. Hay breves datos. biográficos. Viene al final un suplemento (pp. 145-147), un registro de apellidos y una hoja de enmiendas. Dé las 1,200 citaciones sólo 16 son de autotes hispanoamericanos, según Medina.

Ejemplares: $\mathrm{CU} / \mathrm{DLC} / \mathrm{ICJ} / \mathrm{NcU} / \mathrm{NjP} / \mathrm{NNH}$

\section{IGUínIZ, JUAN $\mathrm{B}$.}

12. Catálogo de seudóninos, anagramas e iniciales de escritores mexicanos. Paris, México, Librería de la Vda. de Ch. Bouret, 1913. 62 pp. $23 \mathrm{cms}$.

Se divide el catálogo en dos partes: 1) nombres propios de los autores $(400)$ con sus seudónimos e iniciales, el lugar de nacimiento $y$ de vez en cuando las fechas de nacimiento $y$ de muerte; 2) iniciales $y$ seudónimos seguidos de los apellidos. De apéndice hay un registro de los miembros (18) de la Academia de los Arcades de Roma con los nombres que usaron $y$ un suplemento de 35 seudónimos más.

Ejemplares: DLC/NjP/NN

13. "Seudónimos y anagramas de escritores mexicanos". Biblos. Boletín semanal de información bibliográfica publicado por la Biblioteca Nacional, I, 46 (29 noviembre 1919), 3-4.

Ochenta y nueve seudónimos agregados al Catálogo, seguidos de los nombres propios de los autores.

Ejemplares: $\mathrm{AzU} / \mathrm{C} t / \mathrm{DLC} / \mathrm{TxU}$

14. "Ensayo de un catálogo razonado de obras anónimas y seudónimas de escritores mexicanos". Iguíniz lo cita como inédito en 'su Los historiadores de Jalisco, epitome bibliográfico... México, Oficina Impresora de la Secretaría de Hacienda, Departamento de Comunicaciones, 1918, p. 105. Es de esperar que se resuelva publicar pronto tan útil trabajo. 
LAVERDE AMAYA, IsIDORo.

15. Apuntes sobre bibliografía colombiana, con muestras escogidas en prosa y en verso, por Isidoro Laverde Amaya. Con un apéndice que contiene la lista de las escritoras colombianas, las piezas dramáticas, novelas, libros de historia y de viajes escritos por colombianos. Bogotá, Imp. de Zalamea Hermanos, 1882, viii, 240 pp., 252, iii pp. $21 \mathrm{cms}$.

En las páginas 237-240 se hallan los "seudónimos de colombianos", una lista alfabética de 140 nombres. Al seudónimo sigue el apellido, pero a veces se hallan todos los seudónimos usados por un autor bajo el primer seudónimo puesto por orden alfabético.

Ejemplares: DLC/DPU/MH

\section{López L., Guiliermo.}

16. Indice de seudônimos [chilenos]. [Santiago de Chile], Prensas de la Universidad de Chile, 1939. 108 pp. $25.6 \mathrm{cms}$.

Contiene un prólogo de Raúl Silva Castro, pp. 56-57, además de una lista de "obras consultadas" al principio. Sigue un tegistro de seudónimos - no se incluyen iniciales- por orden alfabético y numerados $(1,224)$; una lista de "firmas y seudónimos de dibujantes y caricaturistas" (38); un índice de antores que hace referencia a los seudónimos por medio de los números; en seguida van los “impresos seudónimos chilenos [113], anteriores a 1926, no incluidos en el Diccionario de anóni'mos y seudónimos hispanoamericanos, de don José 'Toribio Medina", puestos por orden de los seudónimos. Al final hay an indice de nombres propios, pero desgraciadamente no hacen refereneia a la obra seudónima a que corresponden. Por to general es un trabajo fidedigno y merece nuestras alabanzas el autor por haber documentado muy bien cada sendónimo que o él a otro bibliógrafo penetraron.

17. Idem. Anales de la Universidad de Chile, XCVII, 33/34 (10. y 2o, trimestres de 1939), 56-159.

El número 16 es tirada aparte.

Ejemplares: CU/DLC/ICU/NeU/NN/RPB/WaU 


\section{McKnight, WirLIAM A.}

18. Actualmente profesor de la Universidad de North Carolina;

$\because$ tiene en preparación un catálogo de seudónimos de autores españoles.

Maxiriarte, seud. Véase Hartzenbusch é Hiriart, Eugenio, número 11 , arriba.

MEDINA, JosÉ ToRIBIo.

19. Diccionario de anónimos y seudónimos hispanoamericanos. Apuntaciones reunidas por José Toribio Medina. Buenos Aires, Imprenta de la Universidad, 1925. Tomo I, xi, 250 pp., letras A a $\mathrm{H}$. Tomo II, 342 pp., letras I a $Z$ más los indices, $28 \mathrm{cms}$.

Trabajo tirado aparte de la Revista de la Facultad de Filosofía. $y^{-}$Letras, Publicaciones del Instituto de Investigaciones Históricas, números XXVI y XXVI. Hay 3,250 titulos de obras anónimas y seudónimas referentes a los paises iberoamericanos y publicados no importa dónde, desde los tiempos más remotos hasta 1925. Cataloga las obras por orden de los títulos y da a continuación las autoridades que descubrieron el nombre del autor. A veces agrega otras ediciones de la misma obra. En el tomo II pone tres indices que bacen referencia al tomo $y$ a la página donde se balla la obra: 1) registro de iniciales y signoś; 2) de seudónimos: 3) de autores de obras anónimas. Aunque faltan, como lo admite el autor, muchas obras hispanoamericanas, es el catálogo mäs importante y autorizado que tenemos en este campo de la literatura.

Ejemplares: DLC/NN/RPJCB/NcD

MEDINA, José Toribio.

20. "Nuevos anónimos y pseudónimos hispanoamericanos". Obra inédita que cita Guillermo Feliú Cruz bajo el número 405 en la "Bibliografía de D. José Toribio Medina", Boletin del Instituto de Investigaciones Históricas (Buenos Aires), XIII (julio/diciembre de 1931), 220-492.

MOORE, ERNEST RICHARD.

21. Actualmente profesor de Oberlin College; tiene en preparación un catálogo de seudónimos y de publicaciones anónimas 
de autores mexicanos. Suman en la actualidad los seudónimos 2,500 y las obras anónimas o seudónimas 1,500 .

\section{Mostajo, Francisco.}

22. "Contribución al catálogo de pseudónimos". Boletín Bibliográfico publicado por la Biblioteca Central de la Universidad Mayor de San Marcos de Lima, XII, 1/2 (julio 1939), 13-25.

Hay tres grupos: 1) "Pseudónimos de escritores arequipeños" (104) ; 2) "Pseudónimos de escritores de otros departamentos"

( (71) ; 3) "Pseudónimos de extranjeros" que escribieton en el Perú" (10). Agrega el apellido y datos de interés, incluyendo a veces la historia del seudónimo, el título del artículo o del libro, y el nombre del periódico en que apareció. Pero a veces no ha podido descubrir el nombre propio del autor que lo empleó. Añade nna lista de psendónimos de escritores peruanos cuyos nombres ignora (37).

Nocués, José María.

23. "Seudónimos, anónimos, anagramas e iniciales" de autores españoles e hispanoamericanos".

Hacè mención de esta obra inédita José Toribio Medina, que lamenta no haber podido verla (véase el número 19, arriba, tomo I, p. vi). Antes, José Fernández Bremón al escribir el prefacio a la obra de Hartzenbasch (véase el númeto 11, artiba) habia dicho: "Reunidos los materiales para la publicación, Maxiriarth [i. e. Hartzenbusch] tuvo la noticia de baber sido premiada, por la Biblioteca Nacional de Madrid, una obra titulada Seudónimos, anónimos, anagramas e iniciales de autores y traductores españoles e hispanoamericanos, por D. José María Nogués, autor dramático, y segundo Jefe de la Biblioteca de Palacio", p. x. Dados el puesto que ocupó el autor y el honor que recibió, de seguro que contiene datos de gran interés para los bibliógrafos y bibliotecatios. Estamos haciendo gestiones para que se publique, pero hasta la fecha no se ban realizado nuestros deseos.

Patya, TANCREdo de Barros.

24. Achêgas a um diccionario dé pseudonymos, iniciaes, abreviaturas e obras anonymas de auctores brasileiros $e$ de estran- 
geiros, sobre o Brasil ou no mesmo inpressas. Rio de Janeiro, Editores, J. Leite e C $\mathrm{C}^{\mathrm{a}}, 1929.248$ pp. $23.5 \mathrm{cms}$.

Hay cerca de 1,532 citaciones. En la primera de las dos partes se hallà un registro de seudónimos e iniciales por orden alfabético, seguido del título del libro o del artículo, $y$ en este caso, a veces, el nombre de la revista. Ocapan los números 1 a 1,188. Siguen 344 obras anónimas por títulos, juntas con los nombres de los autores. La enumeración continúa hasta el númeto 1.532. A1 final hay un indice de apellidos a los que se agrega el número correspondiente a la obra citada en las dos primeras partes y la ciudad, región o mación de la cual es natural el autor. Ejemplares: DLC/DPU/ICU/IU/MH/NcU/NN

RODRÍGUEZ DEMTORIZI, E.

25. Actualmente director del Archivo de la Nación, Ciudad Trujillo (Santo Domingo), República Dominicana; tiene en preparación un cátálogo de seudónimos dominicanos sacados de periódicos y de libros nacionales.

Rogers, Paul.

26. Tiene casi terminado este colega nuestro en Óberlin College un catálogo de seudónimos de autores españoles tomados de libros y de revistas literarias que se publicaron en España. Suman unos 4,000 ó 5,000 seudónimos, a los que siguen el nombre del autor y breves datos que precisan el periodo de su labor literaria.

Rolando, Carlos A.

27. "Pseudónimos de escritores nacionales y extranjeros en la prensa guayaquileña". Boletín de la Sociedad Ecuatoriana de Estudios Históricos (Quito), III (1919), 273-275.

Véanse las obras siguientes, en las que aumenta el númeto de seudónimos.

28. Cronología del periodismo ecuatoriano. Pseudónimos de la prensa nacional, por Carlos A. Rolando... Guayaquil, Imp. i Papeleria Mercantil Monteverde y Velarde, 1920. 166 pp. $18 \mathrm{cms}$. 
Ocupa la cronologia las primeras 86 páginas y le sigue la sección de seudónimos $(1,250)$. Cataloga éstos por orden alfabético de los nombres propios de los antores; van en seguida el seudónimo, el lugar de publicación de la revista y el nombre de ésta. Hace falta un índice alfabético de los seudónimos.

Ejemplares: DLC/DPU/ICJ/NN

29. "Cronología de la prensa del Ecuador y 1,000 pseudónimos de la prensa nacional", por Carlos A. Rolando. Revista do Instituto Historico e Geographico Brasileiro, tomo, especial, Congresso Internacional de Historia da America (1922), volume I... Director Dr. B. F. Ramiz Galvão, Rio de Janeiro, Inprensa Nacional, 1925. La página siguiente da otro título, a saber, "Annaes do Congresso Internacional de Historia da America". Véase vol. I, pp. 705-794.

Después de una cronología de periódicos ecuatotianos que ocupa las páginas 705 a 757, empieza una lista de seudónimos por orden alfabético de los nombres propios de los autores. Hace unas pocas adiciones $y$ correcciones 'a la obra que publicó en 1920 (véase número 28 arriba).

30. "Pseudónimos en la prensa del Ecuador". Botetín del Centro de Investigaciones Históricas, III, 111 (1933), 208-231.

La misma lista, un poco aumentada, pues incluye unos 1.100 seudónimos.

31. Cronologia del periodismo ecuatoriano. Psendónimos de la prensa nacional, por Carlos A. Rolando. Guayaquil, Tip. y Lit. de la Sociedad Filantrópica del Guayas, 1934. 87 pp. 19 cms.

A pesar de esfuerzos considerables, no hemos podido ver esta edición, pero es casi seguro que aumente $y$ corrija las anteriores y que no hayácambiado el autor el método de sistematización de los asientos.

Ejemplares: $\mathrm{CDU} / \mathrm{DLC} / \mathrm{NNC}$

Scarone, Arturo.

32. Apuntes para un diccionario de seudónimos y de publicaciones anônimas (contribución al estudio de la bibliografia 
del Uruguay), por Arturo Scarone... Prólogo de Ariosto D. González. Montevideo, Imp. "El Siglo Ilustrado", 1926. 75 pp. 25 cms.

En un alfabeto se agrupan obras anónimas, seudónimas e iniciales, dando un total de 147 apuntamientos numerados. Al final se encuentra un registto de nombres propios que hàcen referencia a los apuntes mediante los números. Este estudio ha sido apartado de la Revista del Instituto Histórico y Geográfico del Uruguay.

Ejemplares: de la revista, MH; del libro DLC/DPU/NN

33. Apuntes para un diccionario de seudónimos y de publicaciones anónimas, por Arturo Scarone... Segunda edición (notablemente aumentada y corregida). Prólogo de Ariosto D. González. Montevideo, Imprenta Nacional, 1934. xvi, 351 pp. $23.5 \mathrm{cms}$. (Publicaciones de la Biblioteca Nacional de Montevideo).

Las páginas 3 a 22 son una "Primera Parte. Obras, folletos. artículos periodísticos, etc., escritos con seudónimo o con iniciales" y dispuestos alfabéticamente por ellos. 540 apuntes marcados cón números. La segunda parte, de obras anónimas, ocupa las páginas [223] a $302 y$ los números 541 a 732 . En la primera sección cada apuntamiento suministra referencias precisas al libro - periódico. Siguen las obras anónimas con un 'renglón que las identifica.

Ejemplares: CSmH/DLC.

URIARTE, José EUGENIO DE.

34. Catálogo razonado de obras anónimas y seudónimas de attores de la Compañia de Jestis pertenecientes, á la antigua. asistencia española con nu apéndice de otras de los mismos dignas de especial estudio bibliográfico. . Madrid, Establecimiento Tip. Sucesores de Rivadeneyra, 1904-1916. 5 tomos. $28.5 \mathrm{cms}$. Tomo I, 1904, xxxii, 527 pp. II, 1905, 615 pp. III, 1906, xii, 651 pp. IV, 1914, vii, 606 pp. V, 1916, xxvii, $653 \mathrm{pp}$.

Una obra maestra $y$ monumental que no ha sido igualada por ningún otro bibliógrafo. Cuando muriỏ Uriarte, en 1909, otro 
jesuíta siguió trabajando para completar el tomo cuarto y los indices del último tomo. Este catálogo es sumamente útil a toda persona que emprenda estudios bibliográficos o críticos relacionados con la producción jesuítica o literaria del periodo desde 1540 hasta 1900, porque registra 7,000 obras de jesuítas españoles impresas en España y fuera de ella. Damos a continuación un índice general de la obra, cuya riqueza lo merece $y$ caya utilidad lo justifica.

Tomo 1: Obras anónimas de autores más o menos averiguados, ketras A a Oye.

II: Idem, $P$ a $Z$, dando un total de 2,311 asientos $n a-$ merados. Contiene además

a) Obras anónimas de autores desconocidos, 23122992.

b) Obras anónimas de autores que, al parecer, son jesuítas, 2993-3300.

c) Obras anónimas de autores que pudieran patecer jesuitas y no lo son, 3301-3354.

d) Obras anónimas de autores que por espacio de tiempo eran jesuitas, 3355-3469.

e) Editores anónimos de obtas ajenas dignas de especial mención, 3470-3690.

III: a) Obras seudónimas de autores más ò menos averiguados. $3691-4587$.

b) Obras seudónimas de autores desconocidos, 4588 . 5214 .

c) Obras seudónimas de autores que por notable espacio de tiempo eran jesuitas, 5215-5234.

IV; a) Obras en que intervinieron autores jesuítas sin tener sus nombres en la portada, 5235-5514.

b) Obras de autores de la Compañia confundidos con otros de su nombre y apellido, 5515-5547.

c) Obras y ediciones maliciosa 0 descuidadamente atribuidas a jesuitas, 5548-5662.

d) Obras que, o no existen o son de dudosa existencia. citadas con nombres de jesuíta, 5663-6085.

e) Obras auténticas de autores jesuitas, sobre cuya legitimidad ha habido o pudiera haber alguna disputa, 6086-6160.

f) Suplemento de obras anónimas y seuđónimas pertenecientes a las diversas secciones de toda la obra, Letras A a LL; números 6161-6587.

V: a) Idem, $M$ a $Z, 6588-6860$.

b) Suplemento segundo, 6861-6966.

c) Adiciones y correcciones a toda la obra, pp. 99. 129.

Sigue un grupo de íadices a las 6,966 obras registradas en los cinco tomos, que son de 
1) las primeras palabras con que comienzan los titulos de todas las obras, pp. 133-216.

2) las obras consultadas, pp. $217-421$.

3) autores, traductores $y$ editores anónimos, $p p$. 422-442.

4) autores seudónimos, pp. 443-476.

5) autores y seudónimos que usatọn, pp. 477-506.

6) todas las obras catalogadas, por otden de materias, pp. 507-622.

7) algunas cosas notables tomadas de las discusiones o notas críticas, pp. 623-628.

8) autores tefutados, pp. 629-638.

9) las imprentas en que se publicaron las obras, por orden de las ciudades donde se las imprimieron, pp. 639-652.

Por el perfecto conocimiento de los materiales que consultaron los dos antores, resulta esta bibliografía la autoridad máxima por los libros de que trata. El método, como se ve, nada ha dejado que desear.

Ejemplares: $\mathrm{CU} / \mathrm{DLC} / \mathrm{ICJ} / \mathrm{NjP} / \mathrm{MH} / \mathrm{NN}$

\section{VICTORICA, RICARDO.}

35. Errores y omisiones del "Diccionario de anónimos y pseu, dónimos hispanoamoricanos" de José Toribio Medina... Buenos Aires, Viau y Zona, 1928. 338 pp. $27.5 \mathrm{cms}$.

Agrapa en un solo alfabeto seudónimos, iniciales, apellidos y títulos de obras anónimas o seudónimas, sirviéndose de un sistema muy molesto de referencias de una parte a otra. Ha podido agregar cosà de 1,500 títulos a la obra de Medina, pero la mayoría no son ni omisiones ni correcciones. Aunque no to explica Victorica, ha agregado obras aparecidas después de 1925, año en que concluyó su obra Medina, además de obras sacadas del catálogo de Barros Arana y de otras bibliografias ajenas que se refieren no solamente a los paises sudamericanos sino tambiến a los norteamericanos $y$ de vez en cuando a los paises enropeos (Véase Anonyms and pseudonyms, obra de Adah V. Morris. Chicago, University of Chicago Press, 1933. pp. 16-19. $\mathrm{Da}$ tha lista de catálogos de anónimos y seudónimos referentes a las literaturas norteameticana, europeas, etc.). Medina, en cambio, limitó la extensión de su catálogo porque se dió cuenta de que ya se habian realizado estudios muy extensos en los otros campos y que al copiatlos nada agregaria a la utilidad de su obra. Sin embargo. cotejando este catálogo y el siguiente con las Advertencias de Feliú Cruz (véase núm. 7, arriba), puede sacar el bibliógrafo prevenido alguno que otro dato de interés y provecho. Al final hay adenda, 
pp. [333] a 336 y corrigenda a la obra de Medina, pp. 336 a" 338.

Ejemplates: DLC/NcU/NN/NNC/RPJCB

36. Nueva Epanortosis al "Diccionario de anónimos y seudónimos" de J. T. Medina... Buenos Aires, L. J. Rosso Editor, 1929. 207 pp. 27 cms.

Con el mismo método antes empleado, agtega otras mil obras seudónimas $y$ anónimas. (Véase número 35, arriba).

Ejemplares: DLC

WALTHER, DON.

37. "Catálogo de seudónimos de autores costarricenses".

Este profesor de 1a. Universidad de North Carolina ha hecho el feliz hallazgo de un catálogo de seudónimos en la Biblioteca Nacional de Costa Rica. Lo había dejado trunco a su muerte un empleado de la misma biblioteca y ahota, gracias a la cortesia de las autoridades, el señor Walther podrá completarlo y publicarlo. Esperamos publicarlo en un número futuro de la REVISTA IBEROAMERICANA.

[ANdRade, Vicente de Paúl].

38. "Seudónimos de escritores". El Tiempo (México, D. F.), XXI, 7050, 16 de abril 1904, p. [1]. Firmado: "Luis B. Munoz" [seud.]:

El primer catálogo de seudónimos de escritores mexicanos. Lista 97 escritores por orden alfabético de apellidos y los sigue con los seudónimos que empleaban. Iguíniz, en su Catálogo (véase número 12, arriba), dejó incluidos estos seudónimos. Existe el original del artículo que publicó Andrade, con algunas correcciones hechas por él de su puño y letra, en la Biblioteca de la Universidad de Tejas.

Machado, Jose E.

39. "Escarceos bibliográficos. Lista de seudónimos y anónimos en la literatura y en la política venezolanas". Boletin de la Biblioteca Nacional (Caracas), I, 5 (1 octubre 1924), pp. 131-134; II, 6 (1 enero 1925), pp. 163-167; no. 7 (1 abril 1925), pp. 
[193]-196; no. 9 (1 octubre 1925), pp. 262-266; no. 10 (1 enero 1926), pp. 299-303; III, 11 (1 abril 1926), pp. 333-336; no. 12 (1 julio 1926), pp. 264-366; V, 19 (1 abril 1928), pp. 587-591.

En la última entrega hace un indice de los 307 seudónimos aparecidos en artículos anteriores y los pone en orden alfabético, seguidos por los nombres verdaderos. Desgraciadamente este indice no hace referencia al lugar donde primero aparecieron en los numerosos artículos el nombre del autor y el seudónimo que empleó. En los primeros 7 artículos el bibliógrafo proporciona numerosos datos acerca de la biografia y bibliografia de cada autọ que menciona. Lástima que no haya seguido un método que ponga en orden este material que, al parecer, presentaba siempre que llegaba a tener nuevos datos. Es el único catálogo de tal clase que se publicó en Venezuela.

SCARone, Arturo.

40. "Diccionario de seudónimos y de algunos anónimos". Boletín de la Biblioteca Nacional (Caracas), II, 8 (1 julio 1925), pp. 225-240.

Son de autores uruguayos. Presenta los seudónimos por orden alfabético y los sigue con datos bibliográficos y biográficos, inclusive de vez en cuando los títulos de revistas, las fechas de aparición del impreso y unos pocos títulos de obras anónimas. Las ediciones posteriores de este catálogo (véanse los números 32 y 33, arriba), lo aumentan y corrigen.

ERNEST RICHARD MOORE, Oberlin College. 
